\title{
A Pandas complex adapted for piRNA-guided transposon silencing
}

Kang Zhao ${ }^{\# 1}$, Sha Cheng ${ }^{\# 2}$, Na Miao"1, Ping Xü1,3, Xiaohua Lu¹, Yuhan Zhang ${ }^{\# 2,4}$, Ming Wang ${ }^{1}$,

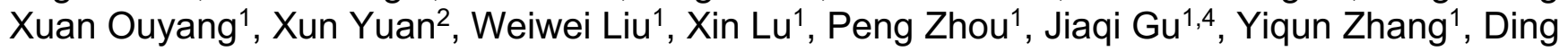
Qiu ${ }^{1}$, Zhaohui Jin², Chen Su${ }^{5}$, Chao Peng ${ }^{5}$, Jian-Hua Wang ${ }^{6}$, Meng-Qiu Dong ${ }^{7,8}$, Youzhong Wan ${ }^{3}$, Jinbiao $\mathrm{Ma}^{4}$, Hong Cheng ${ }^{2}$, Ying Huang ${ }^{2,9}$, Yang $\mathrm{Yu}^{1,9}$

${ }^{1}$ Institute of Biophysics, Key Laboratory of RNA Biology, Chinese Academy of Sciences, University of Chinese Academy of Sciences, Beijing, China, 100101

2State Key Laboratory of Molecular Biology, Shanghai Key Laboratory of Molecular Andrology, CAS Center for Excellence in Molecular Cell Science, Shanghai Institute of Biochemistry and Cell Biology, Chinese Academy of Sciences, University of Chinese Academy of Sciences, Shanghai, China, 200031

${ }^{3}$ School of Life Sciences, National Engineering Laboratory of AIDS Vaccine, Key Laboratory for Molecular Enzymology and Engineering of Ministry of Education, Jilin University, Changchun, Jilin, China, 130012

${ }^{4}$ State Key Laboratory of Genetic Engineering, Department of Biochemistry, School of Life Sciences, Fudan University, Shanghai, China, 200438

${ }^{5}$ National Facility for Protein Science in Shanghai, Zhangjiang Lab, Shanghai Science Research Center, Chinese Academy of Sciences, Shanghai, China, 201210

${ }^{6}$ Graduate School of Peking Union Medical College and Chinese Academy of Sciences of Medical Sciences, Beijing, China, 100730

${ }^{7}$ National Institute of Biological Sciences, Beijing, China, 102206

${ }^{8}$ Tsinghua Institute of Multidisciplinary Biomedical Research, Tsinghua University, Beijing, China, 100084

\#contributed equally

${ }^{9}$ correspondence: yuyang@ibp.ac.cn; huangy@sibcb.ac.cn

Keywords: piRNA, transposon silencing, dNxf2, Panoramix, crystal structure, Drosophila, dNxf1/TAP, dNxt1/p15 


\section{Abstract}

The repression of transposons by the Piwi-interacting RNA (piRNA) pathway is essential to protect animal germ cells. In Drosophila ovaries, Panoramix (Panx) enforces transcriptional silencing by binding to the target-engaged Piwi-piRNA complex, although the precise mechanisms by which this occurs remain elusive. Here, we show that Panx functions together with a germline specific paralogue of a nuclear export factor, dNxf2, and its cofactor dNxt1 (p15), as a ternary complex to suppress transposon expression. Structural and functional analyses demonstrate that $\mathrm{dNxf2}$ binds Panx via its UBA domain, which plays an important role in transposon silencing. Unexpectedly, dNxf2 interacts directly with dNxf1 (TAP), a general nuclear export factor. As a result, $d N x f 2$ prevents $d N x f 1$ from binding to the FG repeats of the nuclear pore complex, a process required for proper RNA export. Transient tethering of $\mathrm{dNxf2}$ to nascent transcripts leads to their nuclear retention. Therefore, we propose that $\mathrm{dNxf2}$ may function as a Pandas (Panoramix-d $\underline{\text { Nxf2 }}$ dependent TAP/p15 silencing) complex, which counteracts the canonical RNA exporting machinery and restricts transposons to the nuclear peripheries. Our findings may have broader implications for understanding how RNA metabolism modulates epigenetic gene silencing and heterochromatin formation. 
64 Transposons are highly abundant in eukaryotes and make up nearly half of the human genome.

65 To maintain eukaryotic genome integrity, nascent transcripts of transposons are often targeted by 66 nuclear Argonaute proteins for transcriptional gene silencing (TGS) ${ }^{1-5}$. In animal gonads, the PIWI-

67 clade Argonautes guided by piRNAs (PIWI-interacting RNA) are thought to recognize nascent 68 transposon transcripts and direct sequence-specific heterochromatin formation ${ }^{1-5}$. As a critical

69 cofactor of Drosophila nuclear Piwi, Panoramix (Panx, also known as Silencio) links the target70 engaged Piwi-piRNA complex to the general silencing machinery ${ }^{6,7}$. Enforced tethering of Panx to 71 nascent transcripts leads to cotranscriptional silencing and correlates with deposition of histone H3 72 lysine 9 trimethylation (H3K9me3) marks ${ }^{6,7}$. However, the mechanism by which Panx mediates this 73 repression remains unknown. An equally important question is why H3K9me3 marks are not always 74 sufficient for transposon silencing ${ }^{8}$. immunoprecipitated with Panx (Extended Data Fig. 1a) and piRNA pathway candidate genes from RNA interference (RNAi) screens ${ }^{9-12}$. Unexpectedly, dNxf2 was identified as a potential cofactor of

79 Panx (Extended Data Fig. 1a-c). dNxf2 belongs to an evolutionarily conserved NXF (nuclear export

81 Instead, dNxf2 and its cofactor dNxt1 (also known as p15) have both been identified in two 82 published RNAi screens as being essential for transposon silencing ${ }^{9,10}$. Like Panx, dNxf2 is 83 specifically expressed in female gonads (Extended Data Fig. 1d).

To validate the interaction between Panx and dNxf2, GFP immunoprecipitation was performed 86 from the ovaries expressing GFP-Panx fusion proteins under its native promoter. Results of mass 87 spectrometry (Extended Data Fig. 1b) and western blot analysis demonstrated that endogenous dNxf2 was associated with Panx (Fig. 1a). Likewise, dNxf2-Halo with a Halo-tag integrated into the 
89 C-terminus was able to precipitate endogenous Panx from Ovarian Somatic Cell (OSC) lysates

90 (Fig. 1b and Extended Data Fig. 6e). Next, we tested whether dNxf2 is functionally required for

91 Panx-mediated silencing. The luciferase transcripts with BoxB sites in their $3^{\prime}$ untranslated regions

92 are repressed if $\lambda \mathrm{N}$-Panx is tethered ${ }^{6,7}$. The expression level of luciferase was measured upon

93 germline-specific knockdowns of either dNxf2 or dNxt1 (Fig. 1c). Despite constitutive tethering of

$94 \lambda \mathrm{N}-$ Panx, loss of either dNxf2 or dNxt1 significantly weakens the ability of Panx to repress the

95 reporter, as compared to the controls (Zuc or attp2, Fig. 1c). Consistent with the reporter

96 derepression, transposon transcripts are elevated upon dNxf2 RNAi (Extended Data Fig. 1e).

97 Taken together, our data suggests that dNxf2 and dNxt1 may function as a heterodimer, either with

98 or downstream of Panx, to suppress transposon expression.

Next, we used RNA sequencing (RNA-seq) to examine global effects on transposon

expected, dNxf2 knockdown triggered a dramatic increase of transposon transcripts (Fig. 1d), similar to that of Panx (Fig. 1e-f), suggesting that dNxf2 is specifically required for silencing of

104 transposons repressed by Panx. To rule out off-target effects of RNAi, a loss of function mutant of $105 \mathrm{dNxf2}$ was generated using CRISPR/Cas9 (Extended Data Fig. 2a) ${ }^{15}$. The dNxf2 mutant female 106 flies carrying a deletion of 20 amino acids at the $\mathrm{N}$-terminus were completely sterile (Extended Data 107 Fig. 2b), similar to other core piRNA pathway mutants ${ }^{6}$. Yet, loss of dNxf2 showed little effect on 108 Piwi nuclear localization or stability (Extended Data Fig. 2c-d), indicating that dNxf2 functions as 109 an effector protein rather than in piRNA biogenesis. Consistent with this notion, the dNxf2 mutants 110 showed global upregulation of transposons (Extended Data Fig. 2e-g) and derepression of the 111 luciferase reporter, despite the $\lambda \mathrm{N}$-Panx tethering (Extended Data Fig. 3a). Unexpectively, absence 112 of dNxf2 noticeably reduces endogenous Panx protein levels (Extended Data Fig. 2d). To rule out 113 the possibility that dNxf2 may indirectly affect transposons via Panx stability, transposon expression 
114 levels were measured upon overexpression of $\lambda \mathrm{N}$-Flag-Panx under the $\mathrm{dNxf2}$ mutant background

115 (Extended Data Fig. 3a-e). Still, the dNxf2 mutant female flies lost transposon controls (Extended

116 Data Fig. 3c-d) and were completely sterile, as if Panx did not exist (Extended Data Fig. 3e).

The striking phenotypic similarities between $\mathrm{dNxf2}$ and Panx prompted us to test whether these

119 two proteins interact directly. We used yeast two-hybrid $(\mathrm{Y} 2 \mathrm{H})$ assays to determine the interacting 120 regions. The domain architecture of $\mathrm{dNxf2}$ is very similar to that of the canonical RNA export factor, 121 dNxf1 (also known as TAP or sbr, Fig. 2a). Both proteins contain leucine-rich repeats (LRR), an 122 RNA recognition motif (RRM), a nuclear transport factor 2 (NTF2)-like domain, and a ubiquitin123 associated domain (UBA). Interestingly, Panx only interacts with the UBA domain of dNxf2 but not 124 that of dNxf1 (Fig. 2b), indicating that this interaction between Panx and dNxf2 is specific (Fig. 2b). 125 Surprisingly, neither the full length nor the NTF2+UBA fragment of dNxf2 could bind Panx (Fig. 2b), 126 suggesting that the UBA domain of dNxf2 might be in a "closed" conformation in the presence of 127 the NTF2 domain. In this regard, the interactions between dNxt1 and the NTF2 domains of either $128 \mathrm{dNxf2}$ or $\mathrm{dNxf1}$ are also weakened in the presence of its UBA domain (Fig. 2c). Since Drosophila 129 Nxt1 itself is absent in the $\mathrm{Y} 2 \mathrm{H}$ system, we tested whether dNxt1 might release the UBA domain 130 from the NTF2 domain to permit Panx binding. Indeed, ectopic expression of dNxt1 is sufficient to 131 allow full length dNxf2 to interact with Panx in a Y2H assay (Fig. 2d). Next, we mapped the minimum 132 region of Panx down to residues $315-343$ (NIR, dN $x$ f2 interacting region) as sufficient for UBA 133 binding (Fig. 2e and Extended Data Fig. 3f). Consistent with the fact that dNxt1 forms a heterodimer 134 with $\mathrm{dNxf2}{ }^{13}$, we found that $\mathrm{dNxt1}$ co-migrates with a fusion protein of $\mathrm{dNxf2}{ }^{\mathrm{NTF} 2+\mathrm{UBA}}-(\mathrm{Gly}-\mathrm{Ser})_{4}$ 135 Panx ${ }^{N I R}$ by size-exclusion chromatography (Fig. 2f), suggesting that Panx, dNxf2, and dNxt1 may 136 exist as a ternary complex. We were not able to crystalize the dNxf2 ${ }^{\text {NTF2 }}$ domain; instead, we 137 crystallized dNxf1 ${ }^{\text {TFF } 2}$ in complex with $\mathrm{dNxt1}$ and determined the structure (Extended Data Fig. 4a). 138 Residues that may be involved in the binding of $\mathrm{dNxf2} 2^{\mathrm{NTF} 2}$ to $\mathrm{dNxt1}$ were modeled according to the 
sequence alignment result and the structure of $d N x f 1^{N T F 2}$ (Extended Data Fig. $4 b$ ). $d N x f 2^{N T F 2}$

140 maintained most, if not all, residues that interact with dNxt1 as validated by the $\mathrm{Y} 2 \mathrm{H}$ and co-

141 immunoprecipitation assays (Fig. 2c and Extended Data Fig. 4a-e), indicating that the interaction

142 mode of $d N x f 2^{N T F 2} / d N x t 1$ complex is like that of $d N x f 1^{N T F 2} / d N x t 1$.

Purified Panx ${ }^{\text {NIR }}$ forms a stable complex with the UBA domain of dNxf2 with a dissociation 145 constant of $\sim 3.2 \mu \mathrm{M}$ as measured by isothermal titration calorimetry (ITC) (Fig. $2 \mathrm{~g}$ ). To further 146 explore the molecular basis of interactions between dNxf2 and Panx, we determined the crystal 147 structure of the dNxf2-Panx complex (Fig. 3a-d). Despite many efforts, only the fusion protein of $148 \mathrm{dNxf2}{ }^{\mathrm{UBA}}-(\text { Gly-Ser)})_{4}-\mathrm{Panx}^{\mathrm{NIR}}$ could successfully be crystallized. The structure was solved at $1.5 \AA$ 149 resolution (Extended Data Table 1). dNxf2 ${ }^{\text {UBA }}$ forms a compact three-helix bundle ( $\left.\alpha 1-\alpha 3\right)$ with a $15033_{10}$-helix (n1) at the C-terminus (Fig. 3b). The Panx ${ }^{\mathrm{NIR}}$ is folded into a long a-helix and lays on the 151 hydrophobic surface formed by $\alpha 2$ and $\alpha 3$ (Fig. 3b-c and Extended Data Fig. 4f). A324, A328, V331, 152 L332, and I335 on Panx interact with V800, F819, F826, F840, L823 and I827 on dNxf2 via 153 hydrophobic interactions (Fig. 3d). Moreover, R321 and R327 on Panx form salt bridges with D837 154 and E799 on $\mathrm{Nxf2}{ }^{\mathrm{UBA}}$, respectively (Fig. 3d). To validate the intermolecular interactions between $155 \mathrm{dNxf2}$ and Panx, key residues on the interacting interface were mutated (Fig. 3e). While either the 156 L823A or D837A single point mutation affected the binding between Panx and dNxf2 ${ }^{\mathrm{BAA}}$, the double 157 point mutation of $\mathrm{dNxf2}{ }^{\mathrm{UBA}}$ (F826A/I827A) nearly abolished its interaction with Panx in both $\mathrm{Y} 2 \mathrm{H}$ 158 and co-immunoprecipitation assays (Fig. 3e-f), highlighting the significant contribution of these 159 residues in Panx binding.

161 In contrast to the highly charged surface of the Nxf1-type UBA (for example human $162 \mathrm{Nxf1/hsNxf1}$ or yeast Mex67/scMex67), dNxf2 UBA favors hydrophobic binding with Panx (Fig. 3c 163 and Extended Data Fig. 5a-b). Key residues on the interacting interface are highly conserved 
164 among different Drosophila species but altered in the Nxf1-type UBA (Extended Data Fig. 5a). On

165 the opposite surface of the Nxf1-type UBA, a hydrophobic pocket is formed to accommodate the 166 FxFG peptide of the nuclear pore complex (NPC) (Extended Data Fig. 5c-d). However, this pocket 167 is missing in dNxf2 ${ }^{\mathrm{UBA}}$ due to a salt bridge formed between K829 and E814 (Extended Data Fig. 168 5c). Additionally, the bulky side chain of L825 on dNxf2 ${ }^{\mathrm{UBA}}$ may hinder FG binding (Extended Data 169 Fig. 5c). In contrast, the corresponding amino acids in hsNxf1 (A602) or scMex67 (G583) contained 170 much smaller side chains (Extended Data Fig. 5c-d), therefore, giving space for FxFG interactions. 171 Consistent with the structural predictions, the dNxf2 ${ }^{\text {UBA }}$ domain was unable to bind to the FG172 repeats of dNup214, a NPC component known to interact with dNxf1 UBA in both Y2H and GST pull 173 down assays (Fig. 3h and Extended Data Fig. 5e) ${ }^{14}$. Similar GST pull down results were obtained 174 using the FG-repeats from dNup358 (Fig. 3h). Since two copies of FG binding domains (NTF2 and 175 UBA) are minimally required for proper RNA export ${ }^{16}$, dNxf2 lacks at least one copy of the FG 176 binding domain (UBA) and thus cannot export RNAs.

To validate the importance of the direct interactions between dNxf2 and Panx in vivo, a previously described $\lambda$ N/BoxB luciferase reporter system ${ }^{6}$ was used to check if artificial tethering 180 of dNxf2 could lead to repression. As expected, significant repression upon tethering of a $\lambda \mathrm{N}-\mathrm{dN} \times \mathrm{f} 2$ 181 fusion protein was observed (Fig. $3 g$ ), unlike that of the negative controls ( $\lambda N$-YFP or GFP-dNxf2 182 lacking a $\lambda N$-tag). Like Panx, the level of $\lambda N$-dNxf2 mediated repression was found in a dosage183 dependent manner, which is correlated with the number of BoxB binding sites (Fig. 3i). Most 184 importantly, the repression is dependent on the presence of the dNxf2 UBA domain (Fig. 3g, dNxf2$\triangle \mathrm{UBA})$.

187 Like dNxf1, dNxf2 contains RNA binding domains (RBDs) at the N-terminus, implying that 188 dNxf2 might directly bind to transposon transcripts (Fig. 3a) ${ }^{13}$. To test this hypothesis, we performed 
189 GoldCLIP (Gel-ommitted Ligation-dependent CLIP)/RT-qPCR experiments, which rely on the 190 covalent attachment of a Halo-tag to its ligand on beads to allow denaturing purification of 191 crosslinked protein-RNA complexes ${ }^{17}$. A Halo-tag was inserted at the C-terminus of dNxf2 (dNxf2192 Halo) using CRISPR/Cas9 (Extended Data Fig. 6a-f) ${ }^{18}$. Mdg1 is one of the transposon families 193 targeted by Piwi/piRNAs in OSCs ${ }^{8,19}$. Strikingly, after UV crosslinking and denaturing washes, the

194 transcripts of mdg1, but not the housekeeping gene rp49, remained attached to the dNxf2-Halo 195 fusion protein (Extended Data Fig. 6b-c). This association depended on UV crosslinking, 196 demonstrating a direct binding between mdg1 and dNxf2 (Extended Data Fig. 6b-c). Interestingly, 197 the interactions were only observed when both histone $\mathrm{H} 1$ and heterochromatin protein 1a (HP1a) 198 were depleted by RNAi, but not in the control knockdown (Extended Data Fig. 6b-c), supporting the 199 idea that the majority of transposon transcripts remain suppressed in a wildtype background. Low 200 steady-state levels of transposon nascent RNAs make it more difficult to obtain significant signals 201 in an already inefficient UV crosslinking experiment. Nevertheless, upon removal of the 202 downstream silencing factors ( $\mathrm{H} 1$ and $\mathrm{HP} 1 \mathrm{a})$ ), transposon transcripts accumulate and are bound 203 by dNxf2-Halo (Extended Data Fig. 6b). In contrast, Frogger, a transposon known not to be targeted 204 by piRNAs ${ }^{19}$, did not show any detectable CLIP signal, although its transcripts are dramatically 205 upregulated upon the H1/HP1a double knockdowns (Extended Data Fig. 6d). This result suggests 206 that the binding of dNxf2 to transposons could be correlated with piRNA targeting ${ }^{19}$. Furthermore, 207 we performed GoldCLIP-seq experiments using dNxf2-Halo knock-in OSCs depleted of Maelstrom, 208 a piRNA pathway effector component either parallel or downstream of $\mathrm{H} 3 \mathrm{~K} 9 \mathrm{me} 3$ establishment on 209 transposons (personal communication with Mikiko Siomi) ${ }^{8,20}$. Consistent with the RT-qPCR results, 210 CLIP-seq data supported the idea that dNxf2 preferentially binds to Piwi-targeted transposons, 211 especially in the absence of Mael (Extended Data Fig. 7). Moreover, the RBDs of dNxf2 are 212 essential for silencing in the tethering assays (Extended Data Fig. 8). Therefore, $\mathrm{dNxf2}^{\mathrm{RBD}}$ is likely 213 to be involved in the effector step of silencing rather than the Piwi-dependent recruitment of dNxf2. 
214 Collectively, our data is consistent with the model that Panx and dNxf2/dNxt1 function together as

215 a stable complex to directly suppress transposons that are targeted by Piwi-piRNAs.

Since loss of Panx leads to a significant reduction of $\mathrm{H} 3 \mathrm{~K} 9 \mathrm{me} 3$ marks on transposons ${ }^{6,7}$, we 218 next tested whether the removal of dNxf2 could result in a similar phenomenon. Considering that 219 Panx is unstable in the absence of dNxf2 (Extended Data Fig. 2d), we performed H3K9me3 ChIP220 qPCR assays over several transposons as well as the Firefly-10xBoxB reporter, while 221 overexpressing $\lambda \mathrm{N}$-Flag-Panx. H3K9me3 showed marginal changes upon removal of $\mathrm{dNxf2.} \mathrm{In}$ 222 contrast, transposon transcripts are still dramatically upregulated in the absence of dNxf2 223 (Extended Data Fig. 3c-d and 9). This result suggested that transposon silencing and H3K9me3 224 deposition could somehow be uncoupled in the dNxf2 mutant. or dNxf2 (Extended Data Fig. 10) ${ }^{21}$. In this regard, I element transcripts, which are targeted by 228 piRNAs, have previously been shown to accumulate within the nucleus ${ }^{22}$. Thus, we proposed that 229 certain RNA export machineries may be regulated by the piRNA pathway to prevent transposon 230 transcripts from being exported out of the nucleus. dNxf1 (TAP) could be one such candidate since 231 hsNxf1 has been reported to dimerize with most NXF family members to regulate RNA export ${ }^{23,24}$. 232 Therefore, we tested whether $\mathrm{dNxf2}$ might interact with $\mathrm{dNxf1}$ and counteract the RNA exporting 233 function of dNxf1. Indeed, GFP-tagged dNxf2 can co-immunoprecipitate Halo-tagged dNxf1 from 234 OSC lysates (Fig. 4a). Either the NTF2 or the UBA domain of dNxf1 is sufficient to interact with $235 \mathrm{dNxf2}{ }^{\mathrm{NTF} 2}$ (Fig. 4b and Extended Data Fig. 11a-c). We noticed here that full length dNxf2 interacts 236 rather weakly with dNxf1 (Fig. 4a), while the interactions seemed much stronger using the truncated 237 versions (Fig. 4b and Extended Data Fig. 11a-c). This implied that the majority of dNxf1 are not 238 available for $\mathrm{dNxf2}$ binding. Consistent with the co-immunoprecipitation results, the interaction 
between dNxf2 and dNxf1 is sufficient to bring together a split Gaussia luciferase, strongly arguing

240 that dNxf2 and dNxf1 can be in close proximity in vivo (Fig. 4c). Furthermore, the GST pull-down

241 assays demonstrated a direct binding between dNxf1 and dNxf2 (Fig. 4d and Extended Data Fig.

242 12a-d). Using chemical crosslinking of proteins coupled with mass spectrometry ${ }^{25}$, we were able to 243 identify key residues in the intermolecular crosslinks within the dNxf1/dNxf2/dNxt1 complex (Fig.

244 4e). We noticed here that K640 and K664 from the UBA domain of dNxf1, sitting on each side of 245 the FG binding pocket, were crosslinked with dNxf2 ${ }^{\mathrm{NTF} 2}$ (Extended Data Fig. 12e). This raised the 246 possibility that $\mathrm{dNxf2}$ binding might block the entry of FG repeats to dNxf1 UBA. In fact, an excess 247 amount of $d N x f 2^{N T F 2}$ can compete $d N x f 1^{\text {NTF2+UBA }}$ off from the FG repeats of dNup214 (Fig. $4 f$ ).

248 Taken together, our results strongly suggest that the dNxf2 ${ }^{\mathrm{NTF} 2}$ domain can directly block the 249 access of the FG binding pockets on either NTF2 or UBA domain of dNxf1. As the binding of dNxf1 250 to NPC FG repeats is essential for RNA export ${ }^{13}$, dNxf2 may inhibit transposon export through 251 blocking dNxf1's ability to bind NPC.

Next, we sought to directly visualize potential transient changes in RNA localization caused by 254 dNxf2, using a rapamycin-inducible tethering system (Fig. 4g and Extended Data Fig. 13). Like any coding transcript, GFP mRNAs containing 10x copies of BoxB binding sites are mostly localized in

256 the cytoplasm despite a constitutive $\lambda \mathrm{N}-\mathrm{FKBP}$ tethering (Fig. $4 \mathrm{~g}$ and Movie S5-12). Upon 257 rapamycin treatment, $\lambda$ N-FKBP dimerizes with the FRB-dNxf2 fusion protein, allowing a transient 258 association of dNxf2 to the GFP mRNAs. Intriguingly, GFP mRNAs start to accumulate at nuclear 259 peripheries upon binding of FRB-dNxf2 (Fig. 4g). The effect is specific to dNxf2 since FRB alone 260 fails to cause any change. Given the involvement of Panx, dNxf2, dNxf1 (TAP) and dNxt1 (p15) in 261 transposon silencing, we named this multi-protein complex as Pandas (Panx-dNxf2 $\underline{\text { dependent }}$ 262 TAPP/p15 s silencing). Our data raised the possibility that deterring the function of dNxf1 in transposon 263 RNA export may be a key event in piRNA-guided silencing (Fig. 4h). In the absence of dNxf2, Panx 
264 fails to efficiently suppress either transposons or the tethered reporters (Extended Data Fig. 3a-e).

265 In this regard, $\lambda N$-Flag-Panx was unable to stay bound to the derepressed transposon chromatin 266 as measured by Flag ChIP-qPCR (Extended Data Fig. 13c). Similarly, the Flag-ChIP signals over

267 the tethered reporter were also diminished even though $\lambda N$-Flag-Panx was constitutively tethered 268 to the RNAs. It is well-established that HP1a can induce heterochromatin formation if tethered via $269 \mathrm{DNA}^{26-29}$. In contrast, direct tethering of HP1a to nascent RNAs fails to do so ${ }^{7}$. Therefore, our data 270 provides a mechanistic insight that sequestering nascent transposon transcripts within the nucleus 271 might be important to fully establish heterochromatin and enforce silencing. By removing dNxf2, we 272 might have uncovered an intermediate state of silencing during heterochromatin formation. prefers to bind the silencing factor Panx rather than the FG repeats of NPCs (Extended Data Fig. 5). Remarkably, we found that dNxf2 can compete with the ability of dNxf1 to bind NPCs (Fig. 4f), thereby preventing RNA export. As dNxf2 preferentially associates with the piRNA-targeted 281 transcripts (Extended Data Fig. 6-7), only a subset of dNxf1 associated with transposons could be 282 affected by dNxf2. Accordingly, part of the silencing function of dNxf2 may be locally hijacking the 283 RNA export machinery and repurposing dNxf1 into a "dead-end" complex, hence trapping 284 transposon transcripts within the nuclear peripheries (Fig. 4h and Extended Data Fig. 13). 285 Interestingly, Dam-ID has shown that both Piwi and NPCs contact chromatin at similar regions ${ }^{30}$. 286 In this regard, dNxf1 has been found to be localized to nuclear peripheries where most constitutive 287 heterochromatin resides ${ }^{13,31-33}$. Our data suggested that sequestering transposons to nuclear 
289 (Extended Data Fig. 10 and Movie 1-4) 32,33 . Intriguingly, Xist can relocate the silenced X290 chromosome to nuclear rim during $\mathrm{X}$ chromosome inactivation in mammalian cells, indicating that 291 similar principles may apply to facultative heterochromatin formation marked with $\mathrm{H} 3 \mathrm{~K} 27 \mathrm{me} 3^{34}$. 292 Recent evidence has demonstrated that hsNxf1 is required for efficient elongation of RNA 293 polymerase $\mathrm{II}^{35}$. It is tempting to speculate that the Pandas complex might also inhibit transcriptional 294 elongation of transposons via neutralizing dNxf1. In summary, we have uncovered an unexpected 295 role of transposon RNA export blockage required for TGS. Our results will have broader 296 implications for understanding how RNA metabolism modulates epigenetic gene silencing and 297 heterochromatin formation (Fig. 4h). 
300

301

302

303

304

305

306

307

308

309

310

311

312

313

314

315

316

317

318

319

320

321

322

323

324

325

326

327

328

329

330

331

332

333

334

335

336

337

338

339

340

341

342

343

344

345

346

347

348

349

\section{REFERENCES}

1. Ge, D. T. \& Zamore, P. D. Small RNA-directed silencing: the fly finds its inner fission yeast? Curr. Biol. 23, R318-20 (2013).

2. Martienssen, R. \& Moazed, D. RNAi and heterochromatin assembly. Cold Spring Harb Perspect Biol 7, a019323 (2015).

3. Czech, B. \& Hannon, G. J. One Loop to Rule Them All: The Ping-Pong Cycle and piRNAGuided Silencing. Trends Biochem. Sci. 0, (2016).

4. Ozata, D. M., Gainetdinov, I., Zoch, A., Carroll, D. X. N. O. X. \& Zamore, P. D. PIWIinteracting RNAs: small RNAs with big functions. Nat. Rev. Genet. 1-20 (2018). doi:10.1038/s41576-018-0073-3

5. Gainetdinov, I., Colpan, C., Arif, A., Cecchini, K. \& Zamore, P. D. A Single Mechanism of Biogenesis, Initiated and Directed by PIWI Proteins, Explains piRNA Production in Most Animals. Mol. Cell 71, 775-790.e5 (2018).

6. $\mathrm{Yu}, \mathrm{Y}$. et al. Panoramix enforces piRNA-dependent cotranscriptional silencing. Science 350, 339-342 (2015).

7. Sienski, G. et al. Silencio/CG9754 connects the Piwi-piRNA complex to the cellular heterochromatin machinery. Genes Dev. 29, 2258-2271 (2015).

8. Sienski, G., Dönertas, D. \& Brennecke, J. Transcriptional silencing of transposons by Piwi and maelstrom and its impact on chromatin state and gene expression. Cell 151, 964-980 (2012).

9. Czech, B., Preall, J. B., McGinn, J. \& Hannon, G. J. A transcriptome-wide RNAi screen in the Drosophila ovary reveals factors of the germline piRNA pathway. Mol. Cell 50, 749-761 (2013).

10. Muerdter, F. et al. A genome-wide RNAi screen draws a genetic framework for transposon control and primary piRNA biogenesis in Drosophila. Mol. Cell 50, 736-748 (2013).

11. Handler, D. et al. The genetic makeup of the Drosophila piRNA pathway. Mol. Cell 50, 762777 (2013).

12. Guruharsha, K. G. et al. A protein complex network of Drosophila melanogaster. Cell 147, 690-703 (2011).

13. Herold, A., Klymenko, T. \& Izaurralde, E. NXF1/p15 heterodimers are essential for mRNA nuclear export in Drosophila. RNA 7, 1768-1780 (2001).

14. Katahira, J. Nuclear export of messenger RNA. Genes (Basel) 6, 163-184 (2015).

15. Port, F., Chen, H.-M., Lee, T. \& Bullock, S. L. Optimized CRISPR/Cas tools for efficient germline and somatic genome engineering in Drosophila. Proc. Natl. Acad. Sci. U.S.A. 111, E2967-76 (2014).

16. Braun, I. C., Herold, A., Rode, M. \& Izaurralde, E. Nuclear export of mRNA by TAP/NXF1 requires two nucleoporin-binding sites but not p15. Mol. Cell. Biol. 22, 5405-5418 (2002).

17. Gu, J. et al. GoldCLIP: Gel-omitted Ligation-dependent CLIP. Genomics Proteomics Bioinformatics 16, 136-143 (2018).

18. Savic, D. et al. CETCh-seq: CRISPR epitope tagging ChIP-seq of DNA-binding proteins. Genome Res. 25, 1581-1589 (2015).

19. Iwasaki, Y. W. et al. Piwi Modulates Chromatin Accessibility by Regulating Multiple Factors Including Histone H1 to Repress Transposons. Mol. Cell 63, 408-419 (2016).

20. Chang, T. H. et al. Maelstrom Represses Canonical Polymerase II Transcription within Bidirectional piRNA Clusters in Drosophila melanogaster. Mol. Cell 73, 291-303.e6 (2019).

21. Radion, E. et al. Key role of piRNAs in telomeric chromatin maintenance and telomere nuclear positioning in Drosophila germline. Epigenetics Chromatin 11, 40 (2018).

22. Chambeyron, S. et al. piRNA-mediated nuclear accumulation of retrotransposon transcripts in the Drosophila female germline. Proc. Natl. Acad. Sci. U.S.A. 105, 14964-14969 (2008).

23. Matzat, L. H., Berberoglu, S. \& Lévesque, L. Formation of a Tap/NXF1 homotypic complex 
is mediated through the amino-terminal domain of Tap and enhances interaction with nucleoporins. Mol. Biol. Cell 19, 327-338 (2008).

24. Aibara, S., Katahira, J., Valkov, E. \& Stewart, M. The principal mRNA nuclear export factor NXF1:NXT1 forms a symmetric binding platform that facilitates export of retroviral CTE-RNA. Nucleic Acids Res. 43, 1883-1893 (2015).

25. Combe, C. W., Fischer, L. \& Rappsilber, J. xiNET: cross-link network maps with residue resolution. Mol. Cell Proteomics 14, 1137-1147 (2015).

26. Danzer, J. R. \& Wallrath, L. L. Mechanisms of HP1-mediated gene silencing in Drosophila. Development 131, 3571-3580 (2004).

27. Hines, K. A. et al. Domains of heterochromatin protein 1 required for Drosophila melanogaster heterochromatin spreading. Genetics 182, 967-977 (2009).

28. Li, Y., Danzer, J. R., Alvarez, P., Belmont, A. S. \& Wallrath, L. L. Effects of tethering HP1 to euchromatic regions of the Drosophila genome. Development 130, 1817-1824 (2003).

29. Azzaz, A. M. et al. Human heterochromatin protein $1 \alpha$ promotes nucleosome associations that drive chromatin condensation. J. Biol. Chem. 289, 6850-6861 (2014).

30. Ilyin, A. A. et al. Piwi interacts with chromatin at nuclear pores and promiscuously binds nuclear transcripts in Drosophila ovarian somatic cells. Nucleic Acids Res. 45, 7666-7680 (2017).

31. Kerkow, D. E. et al. The structure of the NXF2/NXT1 heterodimeric complex reveals the combined specificity and versatility of the NTF2-like fold. J. Mol. Biol. 415, 649-665 (2012).

32. van Steensel, B. \& Belmont, A. S. Lamina-Associated Domains: Links with Chromosome Architecture, Heterochromatin, and Gene Repression. Cell 169, 780-791 (2017).

33. Towbin, B. D., Meister, P. \& Gasser, S. M. The nuclear envelope--a scaffold for silencing? Curr. Opin. Genet. Dev. 19, 180-186 (2009).

34. Chen, C.-K. et al. Xist recruits the $X$ chromosome to the nuclear lamina to enable chromosome-wide silencing. Science 354, 468-472 (2016).

35. Chen, S. et al. The mRNA Export Receptor NXF1 Coordinates Transcriptional Dynamics, Alternative Polyadenylation, and mRNA Export. Mol. Cell 74, 118-131.e7 (2019). 


\section{ACKNOWLEDGEMENTS}

380 We thank the National Facility for Protein Science in Shanghai, Zhangjiang Lab, and Shanghai

381 Science Research Center for their instrumental support and technical assistance. We thank the 382 staff from BL19U1 beamline at the Shanghai Synchrotron Radiation Facility (SSRF) for assistance 383 during data collection. We thank Ms. Shuoguo Li from the Center for Biological Imaging (CBI), 384 Institute of Biophysics, Chinese Academy of Science for her help with taking and analyzing SIM 385 images. This work was supported in part by grants from the Ministry of Science and Technology of 386 China (2017YFA0504200 to Y.Y.) and from the National Natural Science Foundation of China 387 (91640102 and 31870741 to Y.H.; 91640105 and 31770875 to Y.Y.), and the National Postdoctoral 388 Program for Innovative Talents (BX20190081 to Y.H.Z.).

\section{AUTHOR CONTRIBUTIONS}

391 Y.Y. and Y.H. conceived the project and wrote the manuscript. K.Z. constructed the dNxf2392 Halo knock-in OSCs and established the dNxf2 mutant. K.Z., P.X., W.W.L., X.H.L., and 393 D.Q. performed co-immunoprecipitations, tethering assays, transgenic fly constructions, 394 RNA-seq, RT-qPCR experiments. S.C. and X.Y. performed structural studies, beta-gal 395 activity assays, and ITC experiments; S.C. and Y.H.Z. performed GST pull-down assays. K.Z., Z.J., P.Z., X.O., J.G., and P.X. performed cloning. S.C. and X.L. performed Y2H assays. K.Z. and X.O. performed FACS analysis. N.M. preformed FISH and RNAi experiments. M.W. and Y.Q.Z. performed bioinformatics analysis. C.S., C.P., J.H.W., and M.Q.D. performed mass spectrometry and analyzed the data. Y.W., J.M. and H.C. on the manuscript. 
bioRxiv preprint doi: https://doi.org/10.1101/608273; this version posted June 7, 2019. The copyright holder for this preprint (which was not certified by peer review) is the author/funder, who has granted bioRxiv a license to display the preprint in perpetuity. It is made available under aCC-BY-NC-ND 4.0 International license.

\section{COMPETING INTERESTS}

404 The authors declare that there is no conflict of interests.

405

406 MATERIALS \& CORRESPONDENCE

407 All materials and correspondence requesta should be addressed to yuyang@ibp.ac.cn. 
bioRxiv preprint doi: https://doi.org/10.1101/608273; this version posted June 7, 2019. The copyright holder for this preprint (which was not certified by peer review) is the author/funder, who has granted bioRxiv a license to display the preprint in perpetuity. It is made available under aCC-BY-NC-ND 4.0 International license.

\section{Figure 1}

a

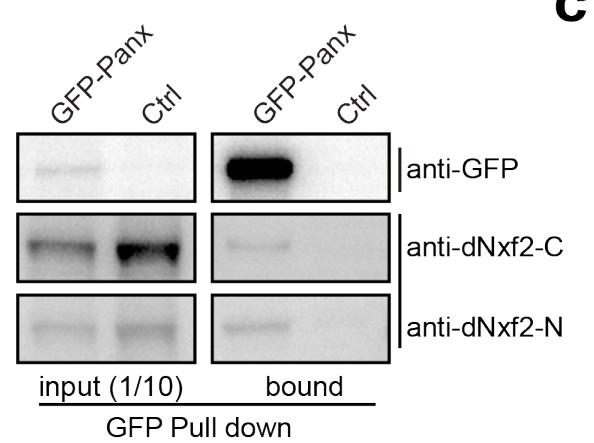

b

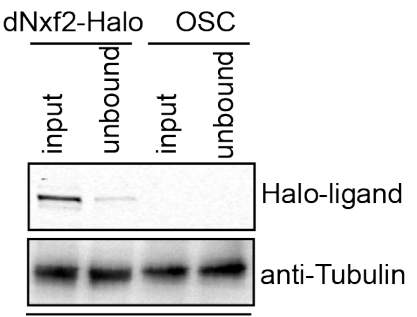

Halo Pull-down

d

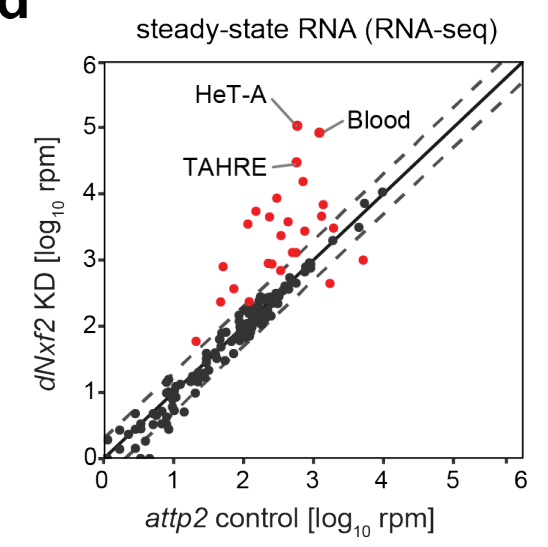

C

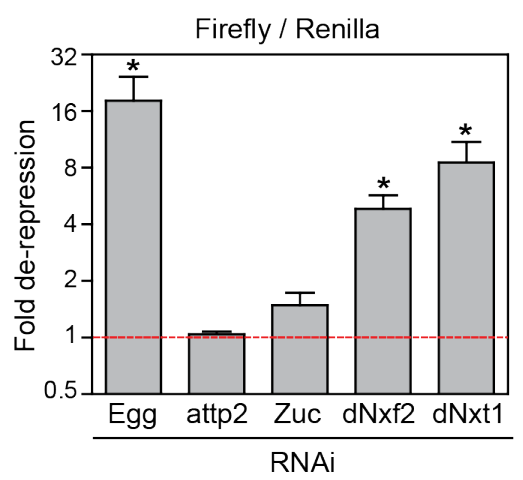

Halo Pull-down

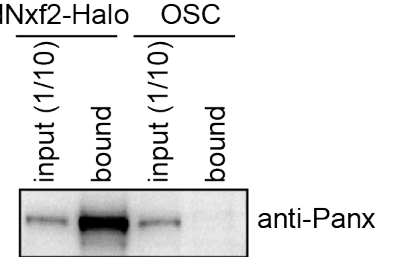

\section{6}

e

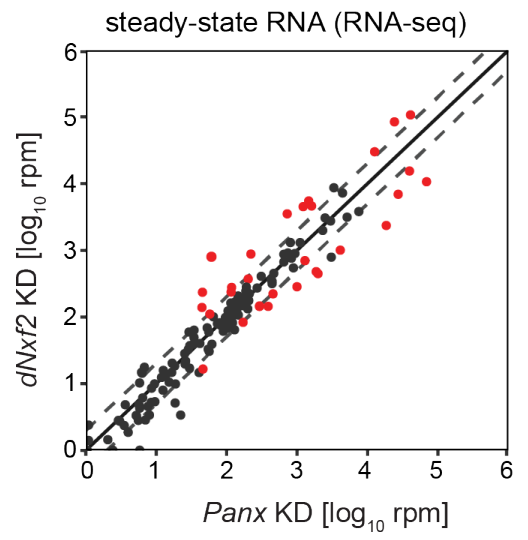

f

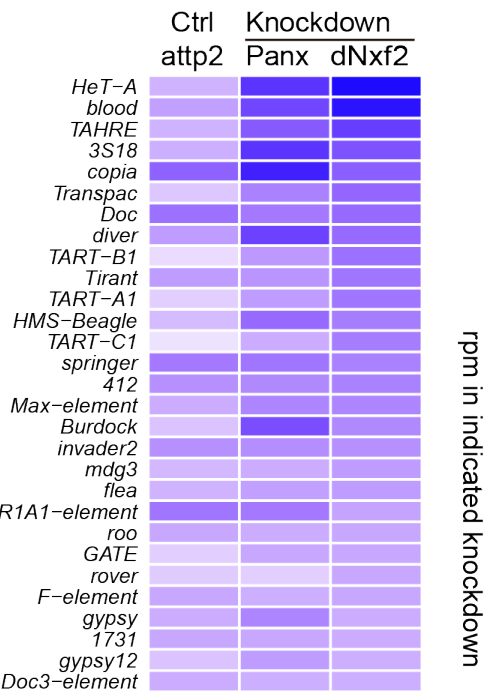

100

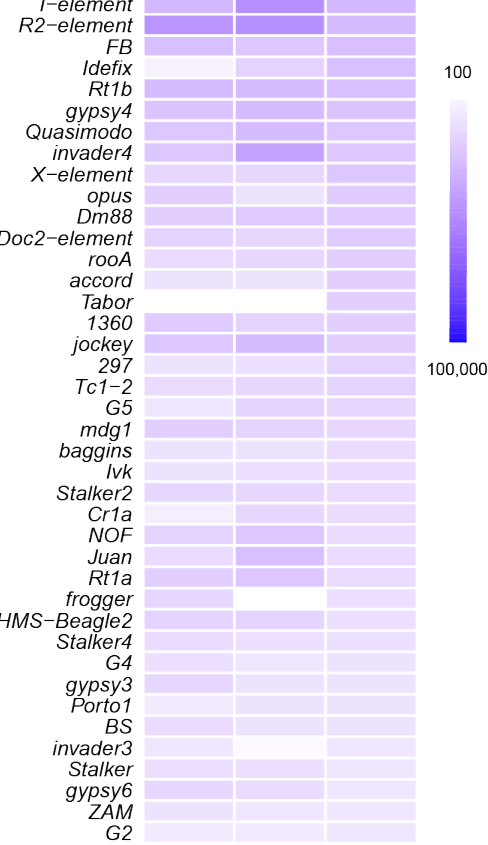


418 Figure 1. dNxf2 functions as a cofactor of Panx in the piRNA pathway.

419 a, Western blots showing co-immunoprecipitation of endogenous dNxf2 with GFP-Panx from ovary 420 lysates. Two different rabbit polyclonal monospecific dNxf2 (dNxf2-N and dNxf2-C) antibodies were 421 used to detect endogenous dNxf2. b, Halo-ligand staining and western blots showing co422 immunoprecipitation of endogenous Panx with dNxf2-Halo driven by the endogenous dNxf2 423 promoter from OSC lysates. A rabbit polyclonal monospecific Panx antibody was used to detect 424 endogenous Panx. The left panel shows depletion of dNxf2-Halo proteins in the unbound sample 425 by Halo beads, and the anti-Tubulin blots serve as loading controls; the right panel shows 426 endogenous Panx proteins. c, The effects of germline (nanos-GAL4) knockdown of the indicated 427 genes on Renilla-normalized Firefly luciferase activity of the reporter while tethering $\lambda \mathrm{N}-\mathrm{Panx}$. For 428 comparison, the relative value of the attp2 control was used for normalization. Data is shown as 429 the mean \pm s.d. $\left(n=15 ;{ }^{*} p=1.41387 \mathrm{E}-07\right)$. $\mathbf{d}$, Comparison of steady-state RNA levels are shown 430 as reads per million (rpm) mapping to the sense strand of each transposon consensus from the 431 nanos-GAL4 driven knockdown for dNxf2 ( $Y$ axis) versus control ( $X$ axis). Dashed lines indicate 432 two-fold changes. The average of two replicates is shown. KD = knockdown. Red dots indicate 433 transposon elements with significant changes. e, Comparison of steady-state RNA levels (RNA434 seq; shown as RPM) mapping to the sense strands of each transposon consensus from the nanos435 GAL4 driven knockdowns of the indicated genes. Red dots indicate transposon elements with 436 significant changes from $\mathbf{d}$. f, Heat map displaying steady-state RNA levels (RNA-seq) as reads 437 per million (rpm) for the top 70 detected transposons from the nanos-GAL4 driven knockdowns of 438 the indicated genes in a blue-white scale. 
bioRxiv preprint doi: https://doi.org/10.1101/608273; this version posted June 7, 2019. The copyright holder for this preprint (which was not certified by peer review) is the author/funder, who has granted bioRxiv a license to display the preprint in perpetuity. It is made available under aCC-BY-NC-ND 4.0 International license.

\section{Figure 2}

a
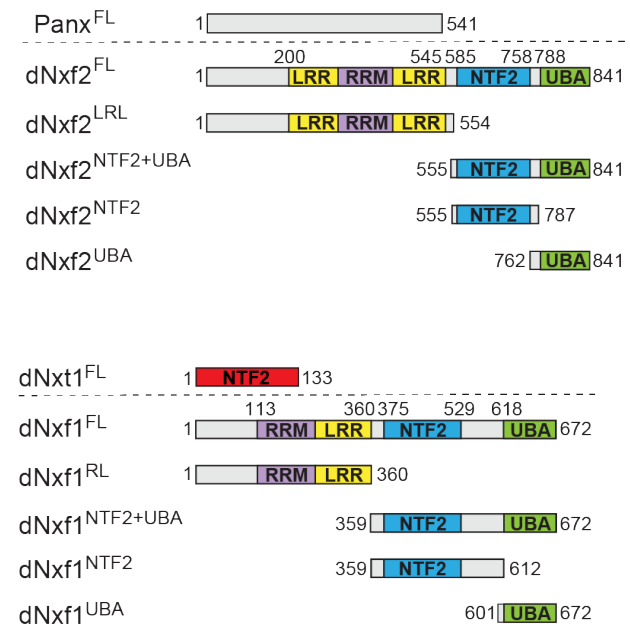
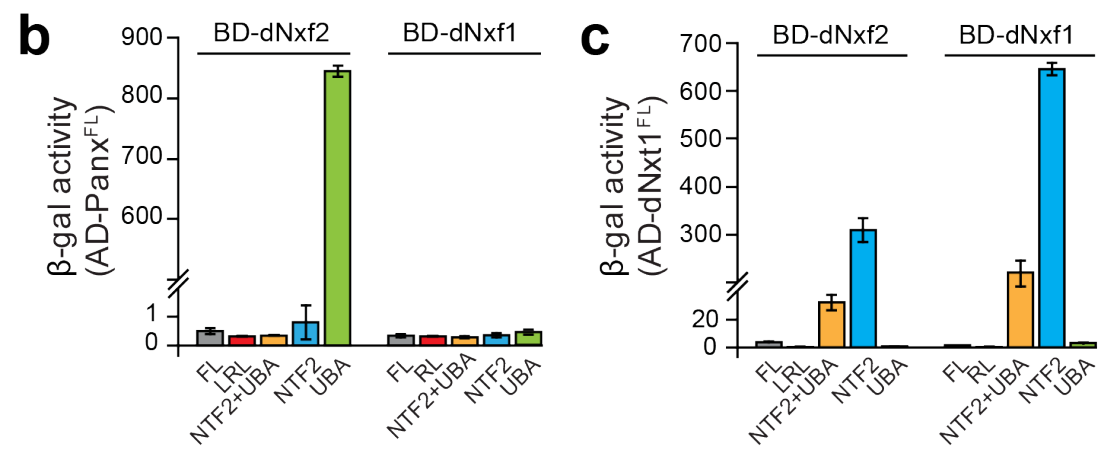

$$
\begin{array}{cccc}
\text { d } & \text { dNxt1 } & \text { BD } & \text { AD } \\
- & d N x f 2 & \times & \text { Panx } \\
+ & d N x f 2 & \times & \text { Panx } \\
+ & \text { YFP } & \times & \text { Panx } \\
+ & \text { dNxf2 } & \times & \text { YFP } \\
- & \text { YFP } & \times & \text { Panx }
\end{array}
$$

YSD-Leu-Trp-Ura-His

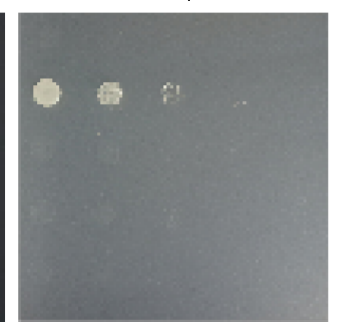

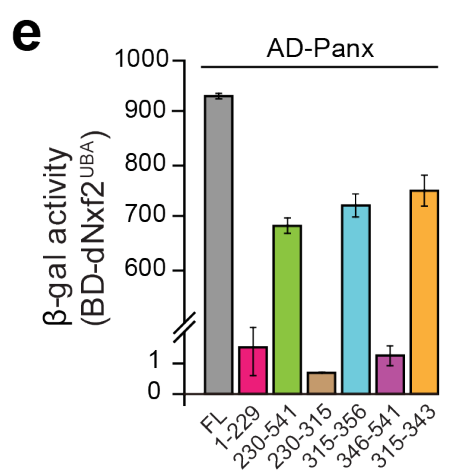

f
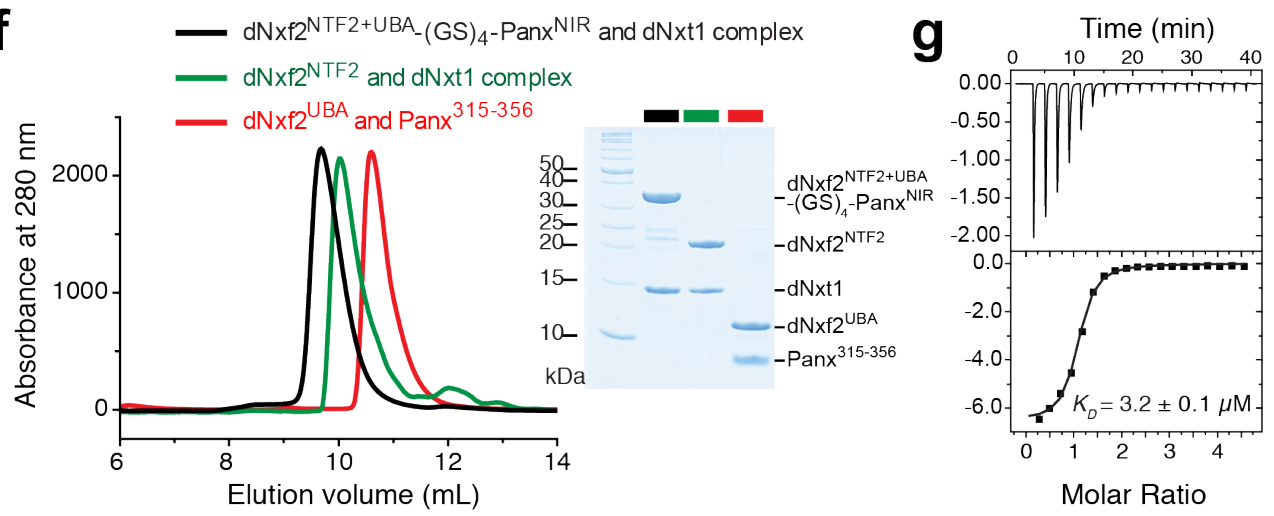


\section{Figure 2. The UBA domain of dNxf2 interacts with Panx directly.}

443 a, Domain architectures of Panx, dNxf2, dNxf1, and dNxt1. Numbers above the diagrams

444 correspond to amino acid residues of each protein. Domain names are abbreviated within

445 respective colored regions. b-e, Yeast two-hybrid $(\mathrm{Y} 2 \mathrm{H})$ assays mapping the interacting regions between Drosophila Nxf1/2 and Panx or dNxt1. Interactions were determined by either measuring

447 the beta-galactosidase activity produced by the reporter gene or growth on YSD media lacking the 448 indicated essential amino acid or uracil. Data are averages of three independent experiments $(n=$ 449 3). Proteins or fragments shown above the dashed line are used as preys in the assays. b, Y2H 450 assays mapping regions of Drosophila Nxf1/2 that interact with Panx. c, Y2H assays mapping 451 regions of Drosophila Nxf1/2 that interact with dNxt1. d, Yeast three hybrid assay determining the 452 requirement of $\mathrm{dNxt1}$ for a Nxf2:Panx interaction. e, $\mathrm{Y} 2 \mathrm{H}$ assays mapping minimum regions of Panx 453 that interact with $\mathrm{dNxf2}{ }^{\mathrm{UBA}}$. $\mathbf{f}$, The left panel shows the size exclusion chromatography profile of the 454 NTF2 and UBA domains of dNxf2 forming heterodimers with dNxt1 and Panx ${ }^{\text {NIR }}$ in solution, 455 respectively. A dNxf2 fragment spanning the NTF2 and UBA domains that is covalently linked to 456 Panx ${ }^{N I R}$ forms a stable complex with dNxt1. The right panel shows the components of the peak in 457 the elution profile by SDS-PAGE. Color schemes used for the three complexes are indicated in the 458 key. g, Quantification of the dissociation constant for the interaction between $\mathrm{dNxf}^{\mathrm{UBA}}$ and Panx $\mathrm{x}^{\mathrm{NIR}}$ 459 as measured by an isothermal titration calorimetry assay. 
bioRxiv preprint doi: https://doi.org/10.1101/608273; this version posted June 7, 2019. The copyright holder for this preprint (which was not certified by peer review) is the author/funder, who has granted bioRxiv a license to display the preprint in perpetuity. It is made available under aCC-BY-NC-ND 4.0 International license.

\section{Figure 3}

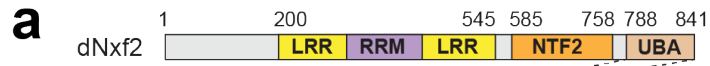

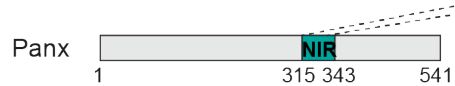

b

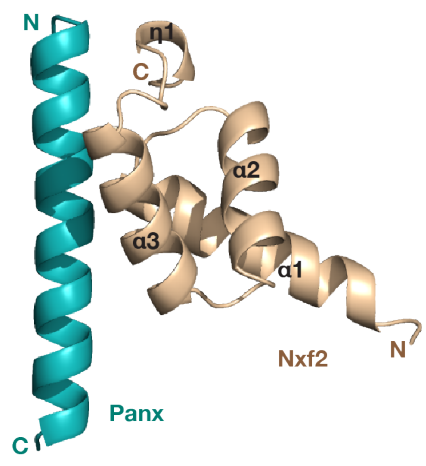

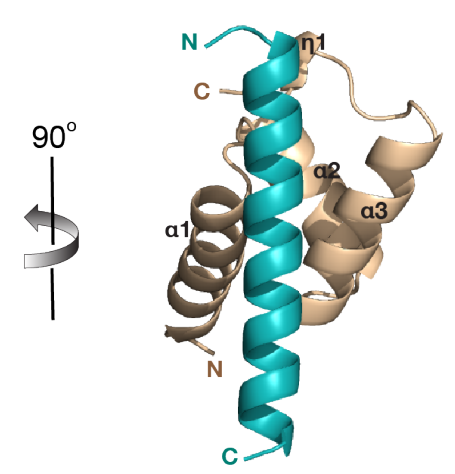

C

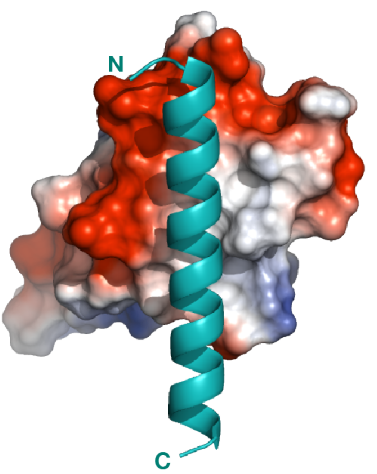

e

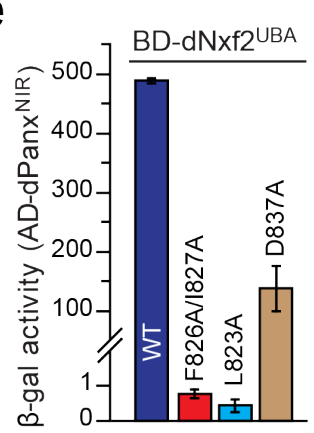

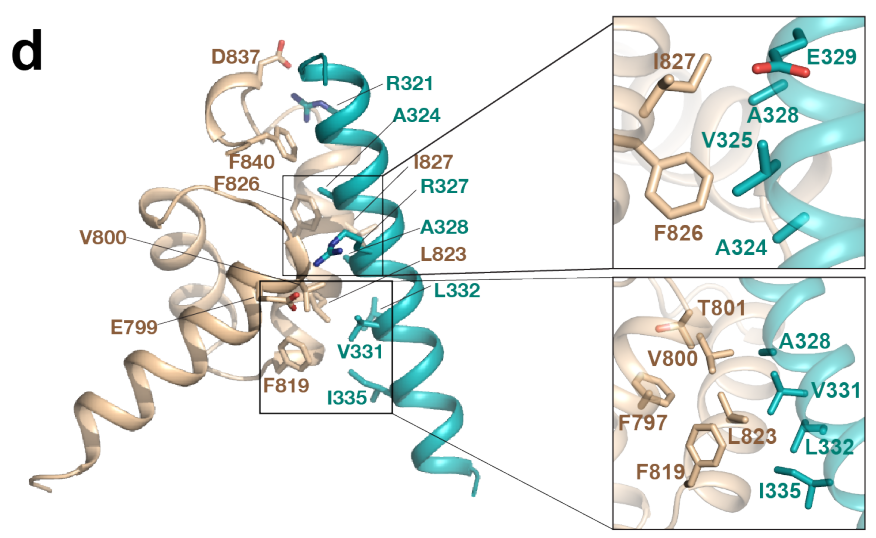

h

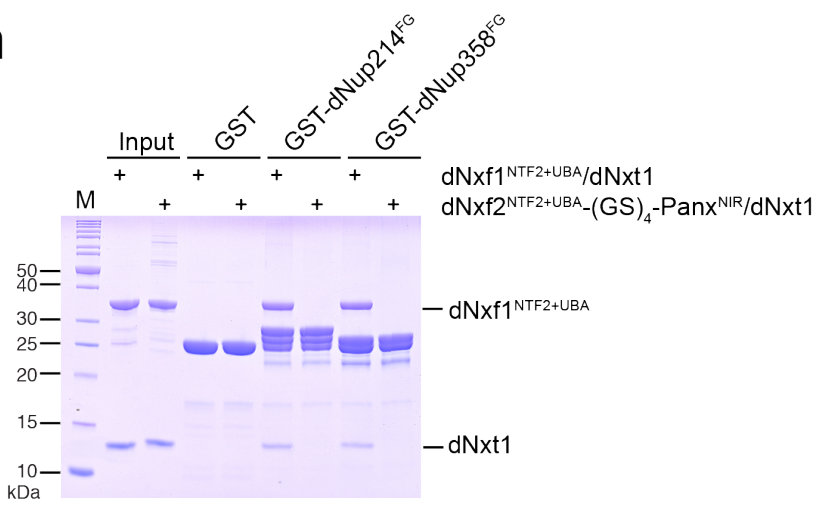

f

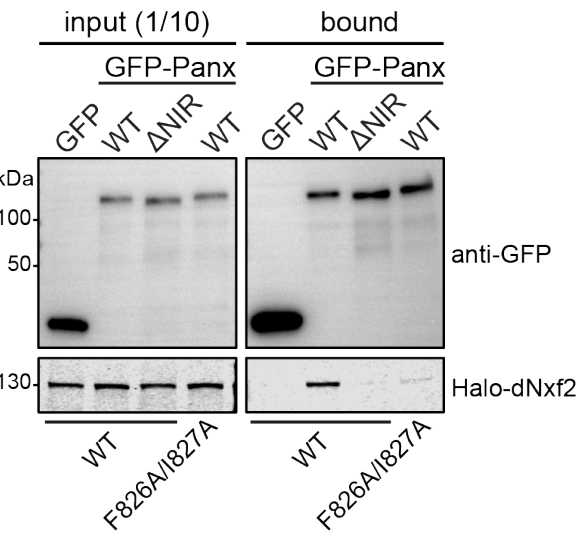

g

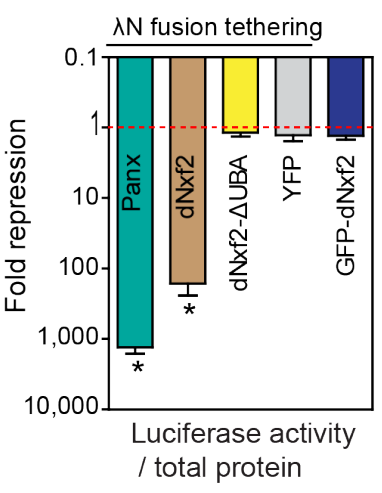

i

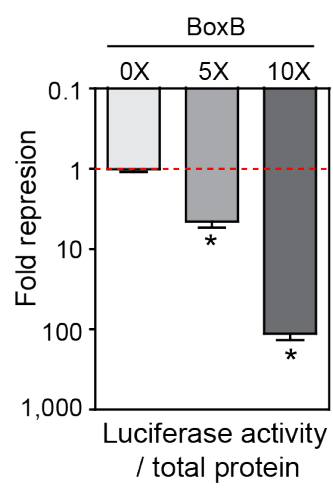


Figure 3. Structure of $\mathrm{dNxf2}^{\mathrm{UBA}}$ in complex with $\mathrm{Panx}^{\mathrm{NIR}}$.

470 a, Schematic of the interacting region between dNxf2 and Panx. Numbers above or below the 471 diagrams correspond to amino acid residues of dNxf2 or Panx, respectively. Domain names are 472 abbreviated within respective colored regions. NIR, dNxf2 interacting region. b, Left, cartoon 473 representation of $d N x f 2^{\mathrm{UBA}}$ in complex with Panx ${ }^{\mathrm{NIR}}$. The $\mathrm{dNxf2}{ }^{\mathrm{UBA}}$ and Panx $\mathrm{NIR}^{\text {fragment are }}$ 474 colored in tan and teal, respectively. Right, a view rotated $90^{\circ}$ around the vertical axis. c, 475 Electrostatic potential analysis of the Panx-binding surface of dNxf2 ${ }^{\mathrm{UBA}}$. Panx ${ }^{\mathrm{NIR}}$ is shown in cartoon mode. $\mathbf{d}$, A detailed view of the interactions between $\mathrm{dNxf} 2^{\mathrm{UBA}}$ and Panx ${ }^{\mathrm{NIR}}$. Key residues involved

477 in binding are shown in sticks. Close-up views of hydrophobic interactions between dNxf2 ${ }^{\mathrm{UBA}}$ and 478 Panx $^{\mathrm{NIR}}$ are shown on the right. e, $\mathrm{Y} 2 \mathrm{H}$ assays measuring the binding of wild-type or mutant $479 \mathrm{dNxf2}{ }^{\mathrm{UBA}}$ with Panx ${ }^{\mathrm{NIR}}$. Mutations of key residues are indicated along the bars. f, Western blots and 480 Halo-ligand staining showing co-immunoprecipitation of GFP-tagged Panx or its NIR deletion 481 mutant $(\triangle \mathrm{NIR})$ with Halo-tagged dNxf2 or its F826A/I827A double mutant from OSC cells. GFP 482 serves as a negative control. $\mathbf{g}$, Effects of the indicated $\lambda \mathrm{N}$ fusion proteins or a non-tethering control 483 (GFP-dNxf2) on luciferase activity of the reporters integrated into the attP2 landing site. Data is 484 shown as the mean \pm s.d. $\left(n=15 ;{ }^{*} p=1.41387 \mathrm{E}-07\right) . \mathbf{h}$, SDS-PAGE showing pull-down results of 485 the $d N x 1^{N T F 2+U B A} / d N x t 1$ complex and dNxf2 ${ }^{N T F 2+U B A}-(G S)_{4}-P a n x^{N I R} / d N x t 1$ complex by either GST486 tagged $\mathrm{dNup} 214^{\mathrm{FG}}$ or dNup358 ${ }^{\mathrm{FG}}$, respectively, compared to a GST control. The beads were 487 washed and aliquots of the bound fraction (20\%) were analyzed. $2 \mu \mathrm{g}$ of each input protein was 488 loaded. Positions of molecular weight markers are indicated on the left in kDa. i, The effects of $\lambda \mathrm{N}$ 489 dNxf2 tethering on luciferase activity of reporters with increasing number of BoxB sites. All reporters 490 are integrated into the same genomic locus (attP2 landing site). Fold repression is calculated as 491 total protein-normalized Firefly luciferase luminescent values of the control (no tethering) divided 492 by that of the indicated experiments. Data is shown as the mean \pm s.d. $\left(n=15 ;{ }^{*} p=1.41387 \mathrm{E}-07\right)$. 
bioRxiv preprint doi: https://doi.org/10.1101/608273; this version posted June 7, 2019. The copyright holder for this preprint (which was not certified by peer review) is the author/funder, who has granted bioRxiv a license to display the preprint in perpetuity. It is made available under aCC-BY-NC-ND 4.0 International license.

\section{Figure 4}

a

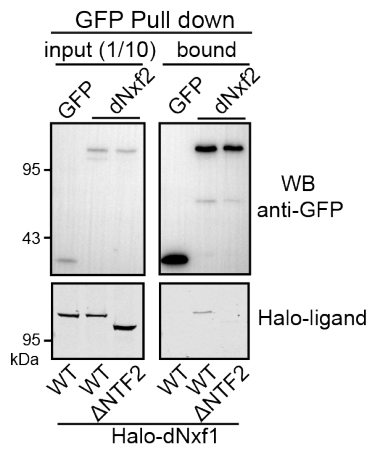

C

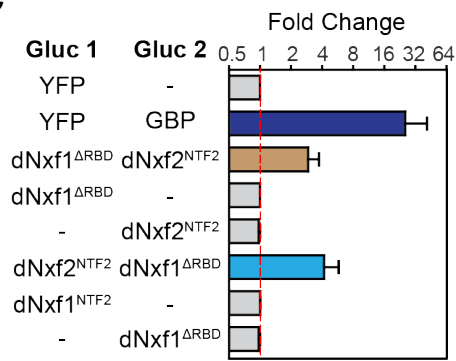

b

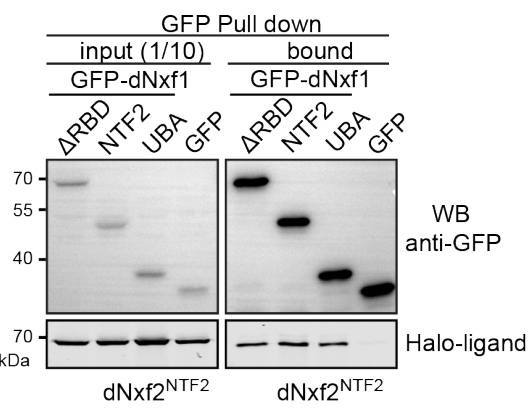

e

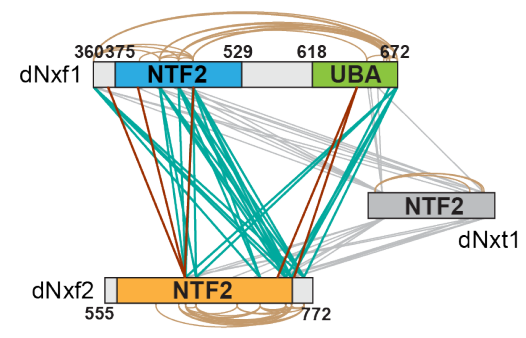

f d
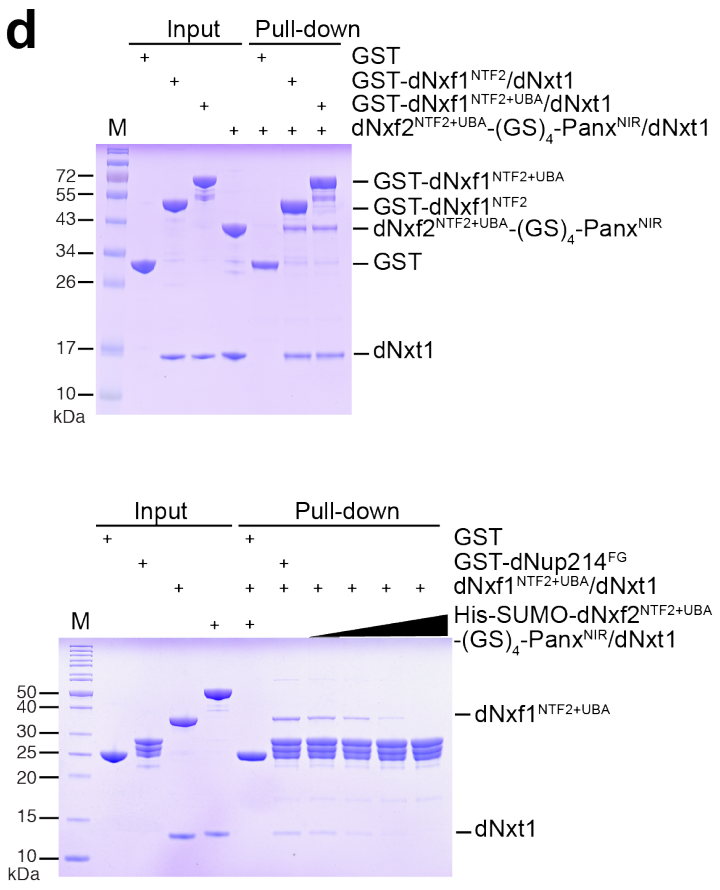

g

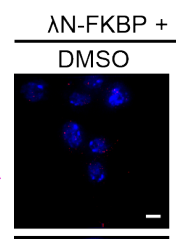

DNA

GFP

protein

GFP mRNA FISH
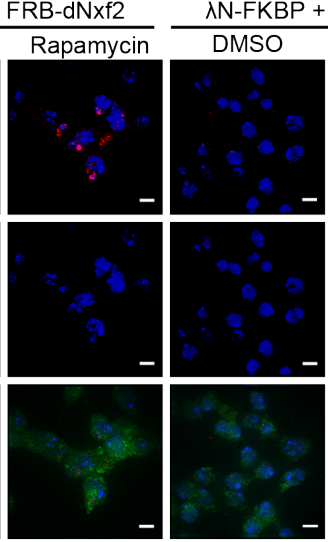

h

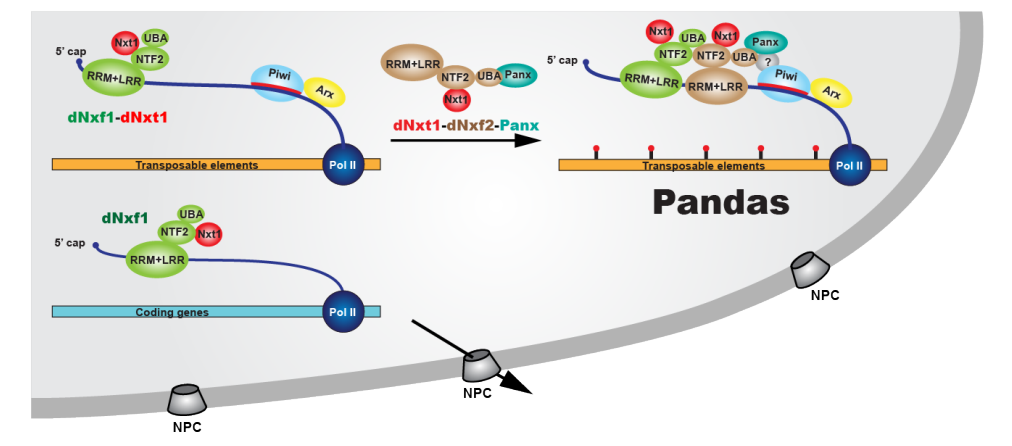

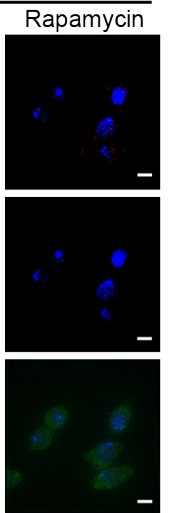


Figure 4. The Pandas complex is required for piRNA-guided transposon silencing.

a, Western blots and Halo-ligand staining showing co-immunoprecipitation of GFP-tagged dNxf2 with Halo-tagged dNxf1 from OSC cells. $\triangle N T F 2$, Halo-dNxf1 lacking the NTF2 domain, and GFP serves as a negative control. b, Western blots and Halo-ligand staining showing coimmunoprecipitation of Halo-tagged dNxf2-NTF2 domain with different domain truncations of GFP-

501 tagged dNxf1 from OSC cells. $\triangle R B D$, dNxf1 lacking the N-terminus RRM and LRR domains; GFP 502 serves as a negative control. c, Split Gaussia luciferase complementation assay results showing 503 for coexpression of the indicated proteins in S2 cells. Fold changes are calculated as total protein 504 normalized luciferase readings divided by that of the corresponding controls. Mean values \pm s.d. 505 from three independent experiments are shown. d, SDS-PAGE showing pull-down results of the $\mathrm{Nxf2}^{\mathrm{NTF2}+\mathrm{UBA}}-(\mathrm{GS})_{4}-\mathrm{Panx}^{\mathrm{NIR}} / \mathrm{dNxt1}$ complex by either GST-tagged $\mathrm{dNxf1}{ }^{\mathrm{NTF}} / \mathrm{dNxt1}$ or $\mathrm{dNxf1}{ }^{\mathrm{NTF} 2+\mathrm{UBA}} / \mathrm{dNxt1}$ respectively, compared to a GST control. The beads were washed and aliquots of the bound fraction (8\%) were analyzed. Each input protein of $2 \mu \mathrm{g}$ was loaded. Positions of molecular weight markers are indicated on the left in kDa. e, Schematic summary of statistical

511 recombinant complex reconstituted in vitro. Intermolecular crosslinks are shown as straight lines in 512 either teal (dNxf1:dNxf2) or grey (dNxt1:dNxf1 or dNxt1:dNxf2) for DSS crosslinking, and in maroon 513 (dNxf1:dNxf2) for EDC crosslinking. Intramolecular crosslinks were shown in brown curves. f, SDS514 PAGE showing the competition assay results of the proteins precipitated by the GST-dNup214FG 515 pre-loaded with the $\mathrm{dNxf} 1^{\mathrm{NTF2}+\mathrm{UBA}} / \mathrm{dNxt1}$ complex, incubated with an increasing amount of the $516 \mathrm{dNxf2}{ }^{\mathrm{NTF2}+\mathrm{UBA}}-(\mathrm{GS})_{4}-\mathrm{Panx} \mathrm{NIR}^{\mathrm{N}} \mathrm{dNxt1}$ complex. The beads were washed and aliquots of the bound 517 fraction (20\%) were analyzed. $2 \mu \mathrm{g}$ of each input protein was loaded. Positions of molecular weight 518 markers are indicated on the left in kDa. g, SIM super-resolution microscopy of RNA FISH. 519 Comparison of the localizations of the reporter mRNAs tethered with $\lambda \mathrm{N}-\mathrm{FKBP}$ upon transient 
521 FKBP:FRB dimerization. DMSO serves as a control. Top panel, RNA signal (red) with DAPI staining; 522 middle, DAPI staining (blue); bottom, RanGAP-GFP fusion protein (green). The scale bars 523 represent $5 \mu \mathrm{m}$ in length. $\mathbf{h}, \mathrm{A}$ model for the Pandas (Panoramix-dNxf2 dependent T사/p15 524 silencing) complex in piRNA-guided silencing, preventing nascent transposon RNA export when 525 targeted by Piwi:piRNAs. 